\title{
Influence of Material on Wheelchair Vibrations ${ }^{\dagger}$
}

\author{
Takeshi Waga *, Soichiro Ura *, Masahito Nagamori *, Hisashi Uchiyama * and Akira Shionoya * \\ Information and Management System Engineering, Nagaoka University of Technoogy, \\ Nagaoka 940-2188, Japan \\ * Correspondence: s195047@stn.nagaokaut.ac.jp (T.W.); ura.soo3635@gmail.com (S.U.); \\ nagamori@kjs.nagaokaut.ac.jp (M.N.); utiyama@vos.nagaokaut.ac.jp (H.U.); \\ shionoya@vos.nagaokaut.ac.jp (A.S.); Tel.: +8180-3329-9922 (T.W.) \\ + Presented at the 13th conference of the International Sports Engineering Association, Online, \\ 22-26 June 2020.
}

Published: 15 June 2020

\begin{abstract}
Wheelchair sports have a tendency to depend on the performance of wheelchairs, and the weight reduction of wheelchairs made of various alloys has helped improve the performance of players. Some players have mentioned, however, that the operability and riding comfort of competition wheelchair have been affected by changing the wheelchair materials; stiffness and weight are considered to be related to operability and riding comfort. In this experiment, we installed some weights on the center of the mass of a competitive wheelchair made of magnesium alloy to be the same mass of a wheelchair made of aluminum alloy; vibrations that occurred on both wheelchairs while driving were measured and compared. The experiment was performed using 3axis sensors. This experiment showed that the vibration frequency of the wheelchair made of magnesium alloy was different from that made of aluminum alloy. This result was thought to be influenced by the difference in Young's modulus or the specific weight.
\end{abstract}

Keywords: competition wheelchair; vibration; stiffness

\section{Introduction}

The holding of the Tokyo Paralympics will be decided in 2020, and competition wheelchair research and development have been conducted to win many medals in Japan. Wheelchair sports have a tendency to depend on the performance of wheelchairs, and the weight reduction of wheelchairs made of various alloys has helped improve the performance of players. Some players have mentioned, however, that the operability and riding comfort of competition wheelchairs have been affected by changing the wheelchair material, and stiffness and weight are considered to be related to operability and riding comfort.

According to a study by Maeda et al., humans may feel uncomfortable due to vibrations when riding a wheelchair on a pedestrian pathway [1]. This is also considered in competitive wheelchair driving.

The purpose of this study is to examine vibrations caused by wheelchair materials. The general competitive wheelchair is made of Aluminum alloy, and new competitive wheelchairs are made of Magnesium alloy. We targeted wheelchairs made from these two alloys. Also, competitive wheelchairs made of magnesium alloy were used in the Rio de Janeiro Paralympics in 2016.

According to Equation (1), the natural frequency decreases as the weight increases. We have prepared a magnesium alloy wheelchair with a weight that is the same weight as an aluminum alloy wheelchair. We observed a vibration that eliminated the weight factor. 


$$
\omega n=\sqrt{\frac{\mathrm{k}}{\mathrm{m}}}
$$

$\omega \mathrm{n}=$ natural frequency; $\mathrm{k}=$ Spring constant; $\mathrm{m}=$ weight.

\section{Experimental Method}

\subsection{Competition Wheelchair}

Figures 1 and 2 show the wheelchairs made of aluminum alloy (Figure 1) and magnesium alloy (Figure 2). In this study, we also prepared a wheelchair with a weight installed to the axle, which is the center of gravity of the wheelchair made of magnesium alloy. This wheelchair is the same weight as the wheelchair made of aluminum alloy. Figure 3 shows how the weight is installed on the axle. Table 1 shows the weight of the wheelchair.

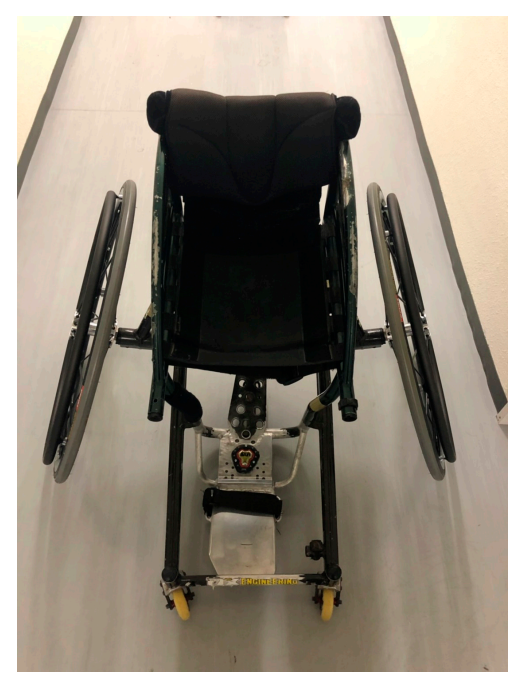

Figure 1. Wheelchair made of aluminum alloy.

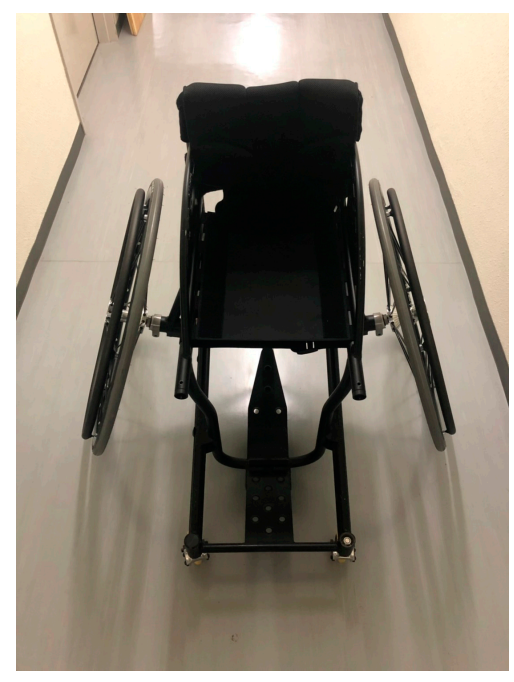

Figure 2. Wheelchair made of magnesium alloy. 


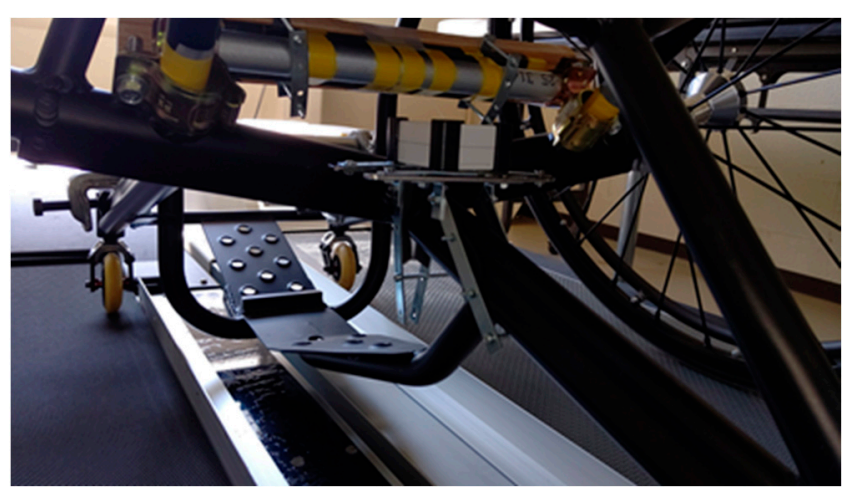

Figure 3 Installed weight.

Table 1. Weight.

\begin{tabular}{ccc}
\hline & Wheelchair Made of Aluminum & Wheelchair Made of Magnesium \\
\hline Weight $[\mathrm{kg}]$ & 12.08 & 10.00 \\
\hline
\end{tabular}

\subsection{Experimental Terms}

Treadmills were used to measure vibration without human movement. The platform was designed to fix the front wheel of a wheelchair on two treadmills. When the treadmills were started using this platform, unmanned driving was possible without the tires idling.

G-MEN-DR100 was used for the accelerometer, and the measurement cycle was $100 \mathrm{~Hz}$ [2].

The wheelchairs were driven on the treadmill for $10 \mathrm{~s}$, and the vibration for the middle $5 \mathrm{~s}$ was measured. Figure 4 shows the experimental scene. The treadmill speed was set to $5 \mathrm{~km} / \mathrm{h}$ or $10 \mathrm{~km} / \mathrm{h}$.

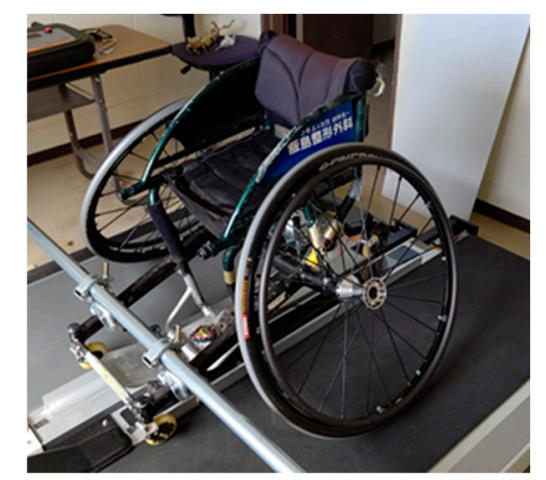

Figure 4. Experimental scene.

The obtained acceleration data were subjected to Fast Fourier Transformanalysis and were normalized using the Hamming window function.

\section{Experiment Result}

\subsection{All Data of Experimental Results}

Tables 2 and 3 show the experimental result. These results were obtained by FFT analysis and were normalized using the Hamming window function of the acceleration data divided by each frequency band. These vibration powers are expressed in $\left[\left(\mathrm{m} / \mathrm{s}^{2}\right)^{2}\right]$, and the total value was obtained. Table 2 shows the $5 \mathrm{~km} / \mathrm{h}$ results, and Table 3 shows the $10 \mathrm{~km} / \mathrm{h}$ results. Among the X, Y, and Z axes, the highest vibration power was $Z$ perpendicular to the treadmill. The vibration power was measured most in the frequency band of $10-20 \mathrm{~Hz}$, followed by $0-10 \mathrm{~Hz}$ and $20-30 \mathrm{~Hz}$. The comparison method was one-way analysis of variance. There was no significant difference in the vibration power between the wheelchairs at $5 \mathrm{~km} / \mathrm{h}$, but at $10 \mathrm{~km} / \mathrm{h}$, a significant difference in the vibration level could be detected depending on the wheelchair. A comparison of the magnesium alloy wheelchair with the 
installed weight and the weightless wheelchair made of magnesium alloy showed that the heavier the wheelchair body, the smaller the vibration power. In the frequency band higher than $20 \mathrm{~Hz}$ at 10 $\mathrm{km} / \mathrm{h}$, the vibration power significantly increased as the weight lessened. A comparison of the wheelchair made of aluminum alloy and the wheelchair made of magnesium with the installed weight showed that the vibration decreased significantly in the $X$-axis direction at $10 \mathrm{~km} / \mathrm{h}$. There was no significant difference between the $\mathrm{Y}$ and $\mathrm{Z}$ axes. It has been clarified that the amount of vibration changes significantly due to material changes in wheelchairs of the same weight.

Table 2. Result of $5 \mathrm{~km} / \mathrm{h}$.

\begin{tabular}{c|c|ccccc}
\hline Wheelchair & axis & $\mathbf{0 - 1 0}$ & $\mathbf{1 0 - 2 0}$ & $\mathbf{2 0 - 3 0}$ & $\mathbf{3 0 - 4 0}$ & $\mathbf{4 0 - 5 0}$ \\
\hline \multirow{3}{*}{$\mathrm{Al}$} & $\mathrm{X}$ & 4.76 & 4.82 & 5.48 & 4.11 & 4.74 \\
& $\mathrm{Y}$ & 9.34 & 7.76 & 4.94 & 3.89 & 6.81 \\
& $\mathrm{Z}$ & $\mathbf{1 0 . 7 5}$ & 18.85 & 6.51 & 5.56 & 5.62 \\
\hline \multirow{3}{*}{$\mathrm{Mg}$} & $\mathrm{X}$ & 5.07 & 3.43 & 4.97 & 4.60 & 6.50 \\
& $\mathrm{Y}$ & 9.66 & 6.07 & 5.32 & 5.48 & 9.63 \\
& $\mathrm{Z}$ & 7.55 & 25.36 & 12.63 & 4.09 & 4.16 \\
\hline \multirow{3}{*}{ Mg installed weight } & $\mathrm{X}$ & 5.65 & 3.69 & 4.86 & 2.94 & 3.55 \\
& $\mathrm{Y}$ & 7.77 & 4.13 & 5.02 & 2.86 & 3.57 \\
& $\mathrm{Z}$ & 8.71 & 23.62 & 7.97 & 4.66 & 4.27 \\
\hline
\end{tabular}

Table 3. Result of $10 \mathrm{~km} / \mathrm{h}$.

\begin{tabular}{ccccccc}
\hline Wheelchair & axis & $\mathbf{0 - 1 0}$ & $\mathbf{1 0 - 2 0}$ & $\mathbf{2 0 - 3 0}$ & $\mathbf{3 0 - 4 0}$ & $\mathbf{4 0 - 5 0}$ \\
\hline \multirow{3}{*}{$\mathrm{Al}$} & $\mathrm{X}$ & 9.41 & 13.67 & 7.88 & 8.06 & 12.94 \\
& $\mathrm{Y}$ & 27.77 & 13.32 & 10.68 & 8.48 & 12.95 \\
& $\mathrm{Z}$ & 21.58 & 74.65 & 15.26 & 15.75 & 18.41 \\
\hline \multirow{3}{*}{$\mathrm{Mg}$} & $\mathrm{X}$ & 6.77 & 7.26 & 7.83 & 13.61 & 14.81 \\
& $\mathrm{Y}$ & 19.78 & 10.15 & 12.65 & 13.00 & 16.11 \\
& $\mathrm{Z}$ & 18.71 & 48.55 & 25.13 & 11.60 & 8.95 \\
\hline \multirow{3}{*}{ Mg installed weight } & $\mathrm{X}$ & 5.04 & 6.51 & 4.62 & 5.54 & 8.88 \\
& $\mathrm{Y}$ & 19.41 & 8.75 & 9.84 & 7.24 & 7.64 \\
& $\mathrm{Z}$ & 17.61 & 52.04 & 17.16 & 7.55 & 8.60 \\
\hline
\end{tabular}

\subsection{Dominant Frequency}

Figure 4 shows the transition of vibration power to observe the dominant frequency on the $\mathrm{Z}$ axis. Table 1 shows the dominant frequencies. At $5 \mathrm{~km} / \mathrm{h}$, dominant frequency of the wheelchair made of aluminum alloy was lower than that of the weightless wheelchair made of magnesium alloy. Furthermore, the dominant frequency of the wheelchair made of magnesium did not change due to a difference in mass.

At a running speed of $10 \mathrm{~km} / \mathrm{h}$, the dominant frequency of the weightless wheelchair made of magnesium alloy was the highest. The dominant frequency of the wheelchair made of aluminum alloy and the installed-weight wheelchair made of magnesium alloy was the same. The dominant frequency of the wheelchair made of aluminum alloy and the installed-weight wheelchair made of magnesium alloy decreased as the driving speed increased. Conversely, the wheelchair made of magnesium alloy increased in dominant frequency with increasing driving speed. 

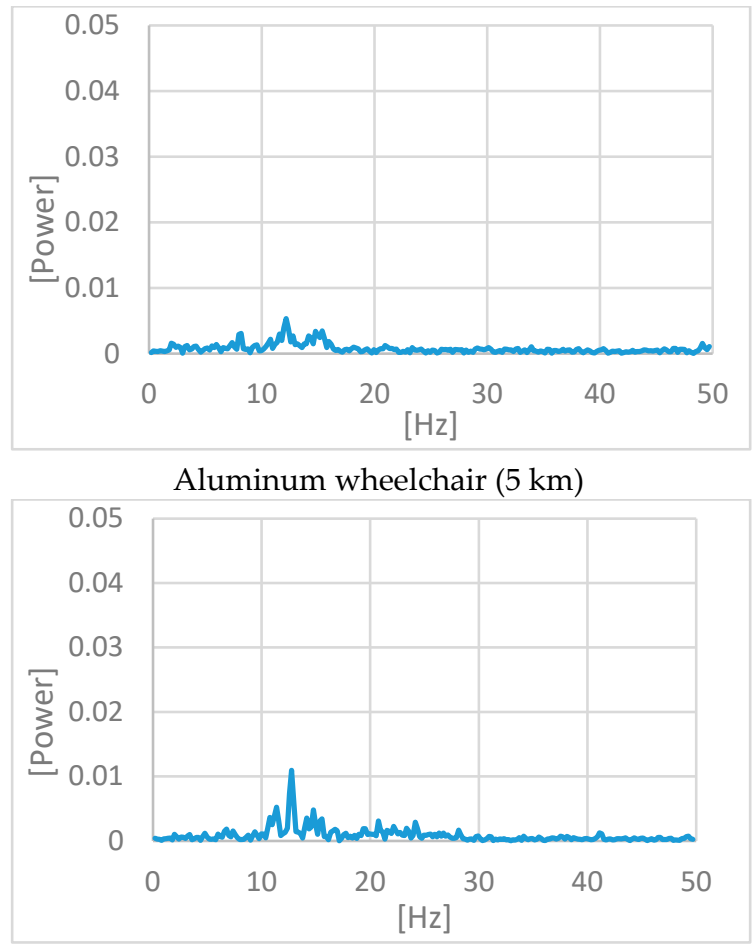

Magnesium wheelchair $(5 \mathrm{~km})$

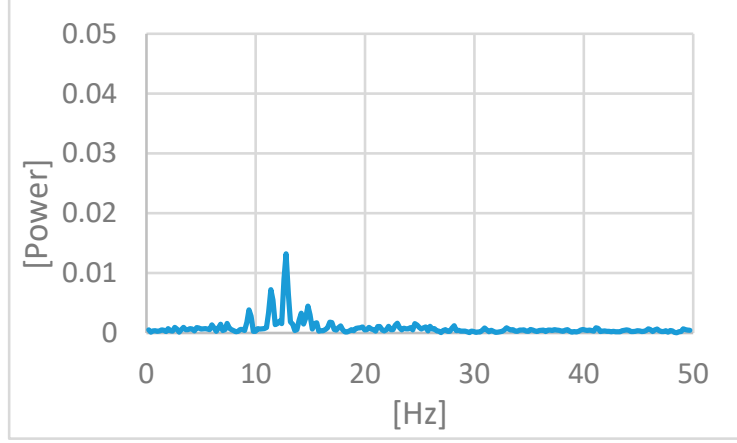

Magnesium wheelchair installed weight $(5 \mathrm{~km})$

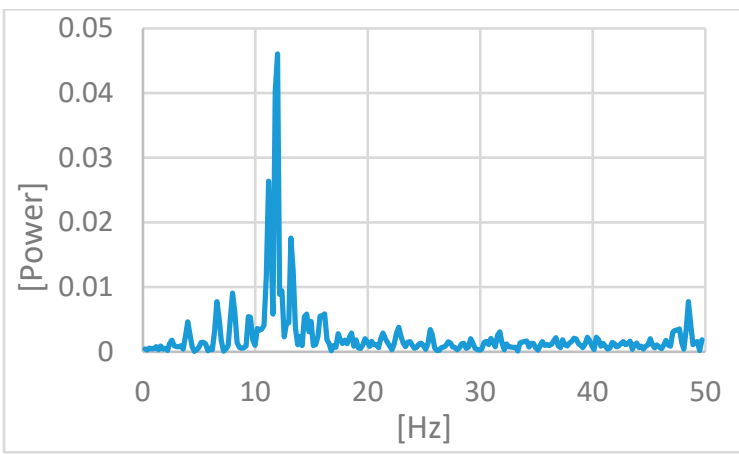

Aluminum wheelchair $(10 \mathrm{~km})$

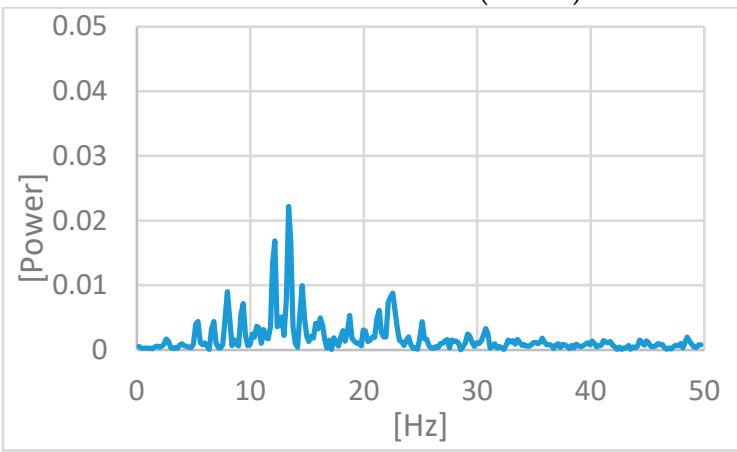

Magnesium wheelchair $(10 \mathrm{~km})$

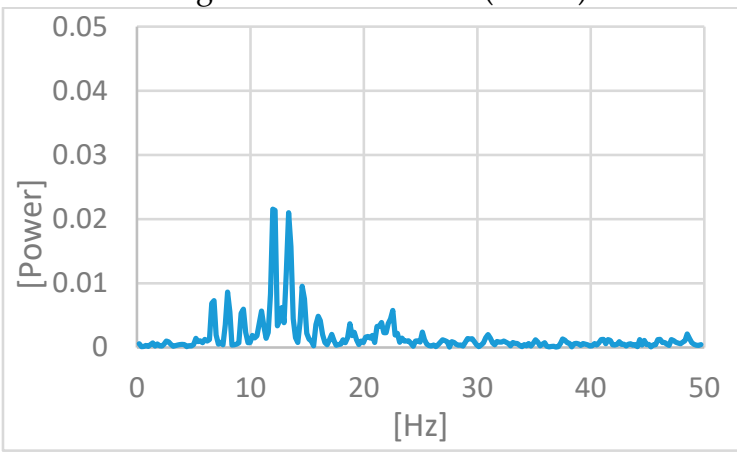

Magnesium wheelchair installed weight $(10 \mathrm{~km})$

Figure 5. Response acceleration spectrum.

Table 4. Frequency of peak value.

\begin{tabular}{ccc}
\hline Wheelchair & $\mathbf{5} \mathbf{~ k m}$ & $\mathbf{1 0} \mathbf{~ k m}$ \\
\hline Aluminum & 12.18 & 11.98 \\
\hline Magnesium & 12.77 & 13.37 \\
\hline Added weight (magnesium) & 12.77 & 11.98 \\
\hline
\end{tabular}

\subsection{RMS of Z-axis Acceleration Waveform}

The RMS (root mean square) is shown in Table 5 . At a driving speed of $5[\mathrm{~km} / \mathrm{h}]$, the RMS of the wheelchair made of magnesium alloy with the installed weight was the largest, but the difference was at most 0.004 . At $10[\mathrm{~km} / \mathrm{h}]$, the RMS of the wheelchair made of aluminum alloy was the highest.

Table 5. RMS of Z-axis acceleration waveform.

\begin{tabular}{ccc}
\hline Wheelchair & $\mathbf{5} \mathbf{~ k m} / \mathbf{h}$ & $\mathbf{1 0} \mathbf{~ k m} / \mathbf{h}$ \\
\hline Aluminum & 0.020639 & 0.106741 \\
\hline Magnesium & 0.023518 & 0.059043 \\
\hline Added weight (magnesium) & 0.025091 & 0.060299 \\
\hline
\end{tabular}




\section{Discussion}

\subsection{Dominant Frequency}

From the results in Table 2, it is considered that the change at $5 \mathrm{~km} / \mathrm{h}$ was caused by the difference in material, and the change at $10 \mathrm{~km} / \mathrm{h}$ was caused by the difference in weight. According to this experiment, it is considered that the difference in the weight of the wheelchair influenced the resonance frequency as stated in the formula above. It can also be inferred that this caused a shift in the sense of operation performance. In the future, it will be necessary to experiment how much a difference in the dominant frequency of $1-2[\mathrm{~Hz}]$ affects the human body. However, since this experiment was performed without a driver, it is necessary to change the vibration characteristics with the driver.

\subsection{RMS}

From the results in Table 3, no difference was found in the vibration acceleration between the material and the weight at a running speed of $5 \mathrm{~km} / \mathrm{h}$. However, the RMS of the wheelchair made of aluminum was greater than two wheelchairs made of magnesium. At $10 \mathrm{~km} / \mathrm{h}$, the wheelchair made of magnesium was considered to have higher absorption of vibration acceleration than the wheelchair made of aluminum.

\section{Conclusions}

Using a treadmill, we verified the vibration characteristics of a wheelchair made of aluminum alloy, a wheelchair made of magnesium alloy, and a wheelchair made of magnesium alloy with installed weight that was the same weight as the wheelchair made of aluminum alloy.

We conducted one-way analysis of variance; when driving at $5[\mathrm{~km} / \mathrm{h}]$, there was no significant difference in vibration. When the treadmill speed was set to $10[\mathrm{~km} / \mathrm{h}]$, all wheelchairs showed an increase in vibration power.

We compared the Z-axis in the driving test at $5[\mathrm{~km} / \mathrm{h}]$; the difference in vibration due to the change in the material was observed, and in the running test at $10 \mathrm{~km} / \mathrm{h}$, the dominant frequency changed due to the change in the weight and the weight of the same material. Based on these findings, it was clarified that the natural frequency changes when the weight changes, which affects the riding comfort.

At $10 \mathrm{~km} / \mathrm{h}$, the wheelchair made of magnesium had less vibration than the wheelchair made of aluminum, confirming the vibration absorption performance [3].

\section{References}

1. Maeda, S.; Futatsuka, M.; Yonesaki, J.; Ikeda, M. Relationship between Questionnaire Survey Results of Vibration Complaints of Wheelchair Users and Vibration Transmissibility of Manual Wheelchair. Environ. Health Prev. Med. 2003, 8, 82-89.

2. G-MEN-DR100. Available online: http://www.g-men.jp/dr/main_gp.html (accessed on 1 August 2019).

3. Li, S.; Li, W.; Li, J. The Application of Magnesium Alloy in Automotive Seat Design. Appl. Mech. Mater. 2013, 395-396. 